\title{
Moral Hazard in Agricultural Yield Insurance
}

\author{
Boyingzi Luo \\ School of Insurance, \\ Central University of Finance and Economics, \\ Beijing, China. P. R.
}

\begin{abstract}
In agricultural yield insurance practices, there are two main categories of insurance products which differed from the targeted insured yield, namely area-yield based insurance and individual-based insurance. A common knowledge is that individualyield based insurance has more flexibility that could meet the real demand of insureds, while having much more severity of moral hazard and higher administration costs. Relatively, area-yield based insurance has lower risk of moral hazard, but obtaining bias, or so-called basis risk at the same time. In this paper, we use an improved modified Miranda Decomposition Model to establish a theoretical framework of farmers behaviors when assuming their goals are to maximize the expected rate of return in agricultural production process under both individual-yield and area-yield insurance. The results show that these two distinct arrangements may cause different motivation to farmers, seducing them act or not act in moral hazard manner.
\end{abstract}

Key words: Moral hazard, Agricultural yield insurance, Miranda Decomposition.

\section{INTRODUCTION}

In many literature([1], [2], [3]), moral hazard is regarded as a very severe problem is agricultural insurance practices. Formally speaking, moral hazard refers to the act and its consequence that occurred when passive actions are taken under high risk situations that could cause unfavorable outcomes, i.e., farmers get loss in their crop yield due to low precipitation. Meanwhile, the argue about the implementation between individual-yield and area-yield insurance has never been stopped. Among all the issues, one thing should be figured out first, that is, how farmers would behave under different arrangements of insurable "yield". Thus, we use some mathematical methods to analyze it throughout this paper.

\section{STATIC MODELS OF MIXED COVERAGE CROP YIELD INSURANCE BASED ON THE MIRANDA DECOMPOSITION}

\section{Theoretical framework}

In this section, we build a mathematical framework depicting the mixed coverage crop insurance based on the Miranda Decomposition. Suppose there are $n$ farmers conducting agricultural productions within a certain area, with $y_{i}, i=1,2, \ldots n$ denoting their individual yields, from which any two are bilaterally linear correlated, and $y_{c}=\sum_{i=1}^{n} w_{i} y_{i}$ denoting the average yield (area yield) with weights $w_{i}, i=1,2, \ldots, n$. Miranda (1991) proposed a famous yield decomposition that

$$
\begin{gathered}
y_{i}-\mu_{i}=\beta_{i}\left(y_{c}-\mu\right)+\epsilon_{i}, \\
\text { where } E\left(y_{i}\right)=\mu_{i}>0, \quad E\left(y_{c}\right)=\mu \text { and } E\left(\epsilon_{i}\right)=0 .(1.1)
\end{gathered}
$$

It actually decomposes the individual yield deviation $y_{i}-\mu_{i}$ into product of a partly correlated $^{1}$ systemic component $y_{c}-\mu$ and a perfectly correlated non-systemic component

\footnotetext{
${ }^{1} \beta_{i}$ could be regarded as a "slope", but not the linear correlation coefficient
} 
$\epsilon_{i}-0$. Particulary, $\beta_{i}=\frac{\operatorname{Cov}\left(y_{i}, y_{c}\right)}{\operatorname{Var}\left(y_{c}\right)}$ is used to describe the sensitivity of individual yields to systemic components for the reason that all systemic risk factors affecting all farmers will be reflected in the deviation $y_{c}-\mu$. It is also assumed that $\operatorname{Cov}\left(y_{c}, \epsilon_{i}\right)=0, \forall i=1,2, \ldots, n$, which infers them to be uncorrelated $\operatorname{Or} \operatorname{Cov}\left(y_{c}, \epsilon_{i} \mid \beta_{i}\right)=0$ which could be verified through (1.1) that indicates $y_{c}$ and $\epsilon_{i}$ are conditionally uncorrelated with respect to $\beta_{i}$ ). Moreover, it is also assumed that the area yield $y_{c}$ and the non-systemic random disturbance $\epsilon_{i}$ both have continuous densities, and are bounded on $\left[0, \max \left(y_{c}\right)\right]$ and $\left[\min \left(\epsilon_{i}\right), \max \left(\epsilon_{i}\right)\right]$ respectively.

With the help of the Miranda Decomposition, the indemnity ${ }^{2}$ of a simple stop loss form crop yield insurance distinctively covering both systemic yield risk and non-systemic yield risk could be expressed as ${ }^{3} I_{i}^{\text {mix }}=n_{c}+n_{i}=\left(x_{c}-y_{c}\right)_{+}+\left(x_{i}-\epsilon_{i}\right)_{+}$, where $x_{c}$ and $x_{i}$ are predetermined indemnity thresholds for systemic and non-systemic yield risk coverage. Moreover, it could be deduced that

$$
\begin{aligned}
\operatorname{Cov}\left(y_{i}, I_{i}^{\text {mix }}\right) & =\operatorname{Cov}\left[\mu_{i}+\beta_{i}\left(y_{c}-\mu\right)+\epsilon_{i},\left(x_{c}-y_{c}\right)_{+}+\left(x_{i}-\epsilon_{i}\right)_{+}\right] \\
& =\beta_{i} \operatorname{Cov}\left(y_{c}, n_{c}\right)+\operatorname{Cov}\left(y_{i}, n_{i}\right)
\end{aligned}
$$

Therefore, if for farmer $i$, the premium for such insurance is $\pi_{i}^{\text {mix }}$ which is deterministic, his cash flow would be $y_{i}^{\text {net }}=y_{i}+I_{i}^{\text {mix }}-\pi_{i}^{\text {mix }}$, and the variance reduction by purchasing insurance would be

$$
\begin{gathered}
\Delta_{i}^{m i x}=\operatorname{Var}\left(y_{i}\right)-\operatorname{Var}\left(y_{i}^{\text {net }}\right) \\
=-\operatorname{Var}\left(n_{c}\right)-\operatorname{Var}\left(n_{i}\right)-2 \operatorname{Cov}\left(n_{c}, n_{i}\right)-2 \beta_{i} \operatorname{Cov}\left(y_{c}, n_{c}\right)-2 \operatorname{Cov}\left(y_{i}, n_{i}\right) .
\end{gathered}
$$

Because $y_{c}$ and $\epsilon_{i}$ are uncorrelated, so that $\operatorname{Cov}\left(n_{c}, n_{i}\right)=0$. Denote $\beta_{c}=-\frac{\operatorname{Var}\left(n_{c}\right)}{2 \operatorname{Cov}\left(y_{c}, n_{c}\right)}$ and $\beta_{d}=$ $-\frac{\operatorname{Var}\left(n_{i}\right)}{2 \operatorname{Cov}\left(y_{i}, n_{i}\right)}=-\frac{\operatorname{Var}\left(n_{i}\right)}{2 \operatorname{Cov}\left(\epsilon_{i}, n_{i}\right)}$, the variance reduction $\Delta_{i}^{\text {mix }}$ thus reducing to

$\Delta_{i}^{m i x}=\operatorname{Var}\left(n_{c}\right)\left(\frac{\beta_{i}}{\beta_{c}}-1\right)+\operatorname{Var}\left(n_{i}\right)\left(\frac{1}{\beta_{d}}-1\right)$, where $\beta_{c} \neq 0, \quad \beta_{d} \neq 0$.

Obviously, the variance reduction in (1.2) depends on the unknown parameters $\beta_{i}, \beta_{c}$ and $\beta_{d}$. In this case, based on sorts of assumptions which fit the reality of agricultural production, we give some lemmas relating to $\beta_{i}$ and $\beta_{d}$ with simple proofs. Note that similar results have been proposed by Miranda in his paper, but without proofs.

Lemma 2.1 If $x_{c}<\mu$ and $x_{i}<0$, then $\beta_{c} \in\left(0, \frac{1}{2}\right), \beta_{d} \in\left(0, \frac{1}{2}\right)$.

Proof. Denote $f(\cdot)$ the density of $y_{c}$, by definition, we have

$$
\begin{aligned}
\operatorname{Var}\left(n_{c}\right)+ & \operatorname{Cov}\left(y_{c}, n_{c}\right)=\int_{0}^{x_{c}}\left(x_{c}-t\right)\left(x_{c}-\mu\right) f(t) d t-\left(\int_{0}^{x_{c}}\left(x_{c}-t\right) f(t) d t\right)^{2} \\
& =\int_{0}^{x_{c}}\left(x_{c}-t\right) f(t) d t\left[x_{c}-\mu-\int_{0}^{x_{c}}\left(x_{c}-t\right) f(t) d t\right]<0 \\
\Rightarrow 0< & \operatorname{Var}\left(n_{c}\right)<-\operatorname{Cov}\left(y_{c}, n_{c}\right) .
\end{aligned}
$$

2 the indemnity is scaled in the yield per insured, without considering the effect of price

$3(x-y)_{+} \triangleq \max (x-y, 0)$ 
In addition, because $\operatorname{Var}\left(\epsilon_{i}\right)=\operatorname{Var}\left[\epsilon_{i}+\min \left(\epsilon_{i}\right)\right]$ and $\operatorname{Cov}\left(\epsilon_{i}, n_{i}\right)=\operatorname{Cov}\left[\epsilon_{i}+\min \left(\epsilon_{i}\right), n_{i}+\right.$ $\left.\min \left(\epsilon_{i}\right)\right]$, a similar form as the above could be implemented which leads to $0<\operatorname{Var}\left(n_{i}\right)<$ $-\operatorname{Cov}\left(\epsilon_{i}, n_{i}\right)$, so the results follow.

Lemma 2.2 Under reasonable distribution assumptions, $\lim _{x_{c} \rightarrow 0} \beta_{c}=0, \lim _{x_{c} \rightarrow \max \left(y_{c}\right)} \beta_{c}=\frac{1}{2}$, $\lim _{x_{i} \rightarrow \min \left(\epsilon_{i}\right)} \beta_{d}=0, \lim _{x_{i} \rightarrow \max \left(\epsilon_{i}\right)} \beta_{d}=\frac{1}{2}$.

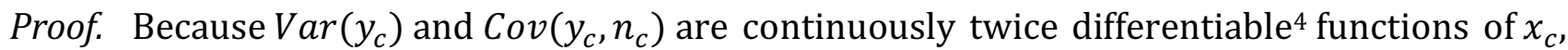
it follows that

$$
\begin{aligned}
& \frac{\partial}{\partial x_{c}} \operatorname{Var}\left(n_{c}\right)=2 x_{c} \int_{0}^{x_{c}} f(t) d t-2 \int_{0}^{x_{c}} t f(t) d t-2 x_{c}\left(\int_{0}^{x_{c}} f(t) d t\right)^{2} \\
& \quad+2 \int_{0}^{x_{c}} t f(t) d t \int_{0}^{x_{c}} f(t) d t \\
& =2\left[\int_{0}^{x_{c}} x_{c} f(t) d t-\int_{0}^{x_{c}} t f(t) d t\right]\left[1-\int_{0}^{x_{c}} f(t) d t\right] . \\
& \frac{\partial}{\partial x_{c}} \operatorname{Cov}\left(y_{c}, n_{c}\right)=\int_{0}^{x_{c}} t f(t) d t-\int_{0}^{x_{c}} \mu f(t) d t \\
& =\left[\int_{0}^{x_{c}} t f(t) d t-\int_{0}^{x_{c}} x_{c} f(t) d t\right]\left[1+\frac{\int_{0}^{x_{c}}\left(\mu-x_{c}\right) f(t) d t}{\int_{0}^{x_{c}} x_{c} f(t) d t-\int_{0}^{x_{c}} t f(t) d t}\right] .
\end{aligned}
$$

Note that in a common sense under agricultural production context, the density $f(\cdot)$ of $y_{c}$ should satisfy $f(0)=0, f\left[\max \left(y_{c}\right)\right]=0, f^{\prime}(0) \neq 0$ and $^{6} f^{\prime}\left[\max \left(y_{c}\right)\right] \neq 0$, thus by taking limits at $x_{c} \rightarrow 0$ and using L Hospital's Rule for twice, we have

$$
\operatorname{Lim}_{x_{c} \rightarrow 0} \frac{\int_{0}^{x_{c}}\left(\mu-x_{c}\right) f(t) d t}{\int_{0}^{x_{c}} x_{c} f(t) d t-\int_{0}^{x_{c}} t f(t) d t}=\lim _{x_{c} \rightarrow 0} \frac{\frac{\partial^{2}\left[\int_{0}^{x_{c}}\left(\mu-x_{c}\right) f(t) d t\right]}{\partial x_{c}^{2}}}{\frac{\partial^{2}\left[\int_{0}^{x_{c}} x_{c} f(t) d t-\int_{0}^{x_{c}} t f(t) d t\right]}{\partial x_{c}^{2}}}=\lim _{x_{c} \rightarrow 0} \frac{\left(\mu-x_{c}\right) f\left(x_{c}\right)}{f\left(x_{c}\right)}-2=\infty .
$$

Consequently, again with L Hospital's Rule, it follows that

$$
\lim _{x_{c} \rightarrow 0} \frac{\operatorname{Var}\left(n_{c}\right)}{\operatorname{Cov}\left(y_{c}, n_{c}\right)}=\lim _{x_{c} \rightarrow 0} \frac{\partial \operatorname{Var}\left(n_{c}\right) / \partial x_{c}}{\partial \operatorname{Cov}\left(y_{c}, n_{c}\right) / \partial x_{c}}=\lim _{x_{c} \rightarrow 0} \frac{-2}{1+\frac{\int_{0}^{x_{c}}\left(\mu-x_{c}\right) f(t) d t}{\int_{0}^{x_{c}} x_{c} f(t) d t-\int_{0}^{x_{c}} t f(t) d t}}=\frac{-2}{\infty}=0 .
$$

On the other hand, as $x_{c}$ approaching to $\infty, n_{c}$ will consistently vary in an opposite manner with any tiny variation of $y_{c}$. In this case, $y_{c}$ and $n_{c}$ will be perfectly negatively correlated, yields their linear correlation coefficient to be -1 , hence we have $\operatorname{Cov}\left(y_{c}, n_{c}\right)=-\operatorname{Var}\left(n_{c}\right)$. As a result, $\lim _{x_{c} \rightarrow \infty} \frac{\operatorname{Var}\left(n_{c}\right)}{\operatorname{Cov}\left(y_{c}, n_{c}\right)}=-1$.

Similar analysis could be implemented to $\epsilon_{i}$ and $x_{i}$, therefore proving the results.

\footnotetext{
4 because all integrands are continuous, so that integrals are differentiable with respect to $x_{c}$

${ }^{5}$ such as general beta distributions with parameters $\alpha>1$ and $\beta>1$, which are often used to fit yield distributions

${ }^{6} f^{\prime}$ denotes the derivative of $f$ with respect to $x_{c}$
} 
Normally, the monotonicity of $\beta_{c}$ with respect to $x_{c}$ is vague because $\frac{\partial \operatorname{Var}\left(n_{c}\right)}{\partial x_{c}}>0$ and $-\frac{\partial \operatorname{Cov}\left(y_{c}, n_{c}\right)}{\partial x_{c}}>0$. Moreover, even for known $f(\cdot)$, the monotonicity is still hard to be verified as it depends on both $x_{c}$ and $f(\cdot)$. Similarly, the same situation happens on $\beta_{d}$ with respect to $x_{i}$. Under this circumstance, we give some sufficient but unnecessary conditions for $\beta_{c}$ increasing with respect to $x_{c}$ and $\beta_{d}$ increasing with respect to $x_{i}$ under different scenarios as convenient and operative tools in examining the monotonicity. In addition, for simplicity and reality, we only consider the cases where $x_{c}<\mu$ and $x_{i}<0$.

Lemma 2.3 Let $F(\cdot)$ and $S(\cdot)$ be the cumulative distribution function and the survival function of $y_{c}$, consider the cases where $x_{c}<\mu$.

1. In the case $S\left(x_{c}\right)>\frac{1}{2}$ and $2 x_{c} S\left(x_{c}\right)>\mu$, if $\int_{0}^{x_{c}} t f(t) d t<\frac{F\left(x_{c}\right)\left[2 x_{c} S\left(x_{c}\right)-\mu\right]}{2 S\left(x_{c}\right)-1}$, then $\beta_{c}$ is increasing with $x_{c}$.

2. In the case $S\left(x_{c}\right)<\frac{1}{2}$, if $\int_{0}^{x_{c}} t f(t) d t>\frac{F\left(x_{c}\right)\left[2 x_{c} S\left(x_{c}\right)-\mu\right]}{2 S\left(x_{c}\right)-1}$, then $\beta_{c}$ is increasing with $x_{c}$.

In the rest cases, whether $\beta_{c}$ is increasing with $x_{c}$ is hard to verify, while all these results fit the similar way for $\beta_{d}$ with $x_{i}$.

Proof. According to Lemma 1.1, if $x_{c}<\mu$, then $0<\operatorname{Var}\left(n_{c}\right)<-\operatorname{Cov}\left(y_{c}, n_{c}\right)$. Denote $-\operatorname{Cov}\left(y_{c}, n_{c}\right)=\operatorname{Var}\left(n_{c}\right)+m$ where $m>0$, we obtain

$$
\begin{gathered}
\frac{\partial\left[\operatorname{Var}\left(n_{c}\right) /-\operatorname{Cov}\left(y_{c}, n_{c}\right)\right]}{\partial x_{c}}=\left\{2 [ \int _ { 0 } ^ { x _ { c } } x _ { c } f ( t ) d t - \int _ { 0 } ^ { x _ { c } } t f ( t ) d t ] [ 1 - \int _ { 0 } ^ { x _ { c } } f ( t ) d t ] \left[\operatorname{Var}\left(n_{c}\right)\right.\right. \\
\left.-\left[\int_{0}^{x_{c}} \mu f(t) d t-\int_{0}^{x_{c}} t f(t) d t\right] \operatorname{Var}\left(n_{c}\right)\right\} /\left[\operatorname{Cov}\left(y_{c}, n_{c}\right)\right]^{2} .
\end{gathered}
$$

Consequently, a sufficient but unnecessary condition for $\frac{\partial\left[\operatorname{Var}\left(n_{c}\right) /-\operatorname{Cov}\left(y_{c}, n_{c}\right)\right]}{\partial x_{c}}>0$ is

$$
2\left[x_{c} F\left(x_{c}\right)-\int_{0}^{x_{c}} t f(t) d t\right]\left[1-F\left(x_{c}\right)\right]>\mu F\left(x_{c}\right)-\int_{0}^{x_{c}} t f(t) d t
$$

Solving this inequality leads to the proof, which is similar to the proof of $\beta_{d}$ with respect to $x_{i}$.

In comparison, as the same as in Miranda's paper, the variance reduction for farmer $i$ by purchasing area-yield crop insurance in which every farmer pays identical premium and gets indistinguishable indemnity ${ }^{7}$ of $I_{i}^{\text {area }}=n_{c}=\left(x_{c}-y_{c}\right)_{+}$will be

$$
\Delta_{i}^{\text {area }}=\operatorname{Var}\left(n_{c}\right)\left(\frac{\beta_{i}}{\beta_{c}}-1\right)
$$

Therefore, according to Lemma 1.1, by comparing (1.2) and (1.3), the following proposition is obtained.

Theorem 2.1 Under full coverage level and reasonable setting of non-systemic indemnity threshold $x_{i}<E\left(\epsilon_{i}\right)=0$, if the systemic indemnity threshold $x_{c}$ is the same as the indemnity threshold in area-yield insurance arrangement, then the mixed coverage crop yield insurance is always more risk reducing (by reducing the variance of net production income) compared with

7 in this case, $I_{i}^{\text {area }}=I_{j}^{\text {area }}, \forall i, j=1,2, \ldots, n$ 
area-yield crop insurance (Or per se, $\Delta_{i}^{\text {mix }} \geqslant \Delta_{i}^{\text {area }}$, with equation holds only when $\beta_{i}=1$, that is, $\operatorname{Var}\left(n_{i}\right)=0$ ).

Meanwhile, if a farmer is free to choose his coverage level, then a procedure of coverage level optimization could be implemented relative to some certain purposes. Specifically, with $\phi_{i}$ denoting the selected coverage level of area-yield crop insurance for farmer $i, \varphi_{i}$ denoting his selected coverage level of mixed coverage crop yield insurance, we have by (1.2) and (1.3)

$\Delta_{i}^{\text {area }}=\operatorname{Var}\left(n_{c}\right)\left(\frac{\beta_{i}}{\beta_{c}} \phi_{i}-\phi_{i}^{2}\right)$

$\Delta_{i}^{m i x}=\operatorname{Var}\left(n_{c}\right)\left(\frac{\beta_{i}}{\beta_{c}} \varphi_{i}-\varphi_{i}^{2}\right)+\operatorname{Var}\left(n_{i}\right)\left(\frac{1}{\beta_{d}} \varphi_{i}-\varphi_{i}^{2}\right)$.

Note that $\frac{\partial \Delta_{i}^{\text {mix }}}{\partial \phi_{i}}<0$ and $\frac{\partial \Delta_{i}^{\text {area }}}{\partial \varphi_{i}}<0$, which infer potential unique global maximums. Therefore, the optimal coverage levels maximizing the variance reductions (1.4) and (1.5) will be

$$
\phi_{i}^{*}=\frac{\beta_{i}}{2 \beta_{c}} \quad \text { and } \varphi_{i}^{*}=\frac{1}{2}\left[\frac{\frac{\beta_{i} \operatorname{Var}\left(n_{c}\right)}{\beta_{c}}+\frac{\operatorname{Var}\left(n_{i}\right)}{\beta_{d}}}{\operatorname{Var}\left(n_{c}\right)+\operatorname{Var}\left(n_{i}\right)}\right] .
$$

Obviously, from (1.6), it could be deduced that $\frac{\beta_{i}}{\beta_{c}}>\frac{1}{\beta_{d}} \Leftrightarrow \phi_{i}^{*}>\varphi_{i}^{*}$. However, the conclusion about which one is larger could only be clarified empirically in terms of the assumptions in (1.1) which infer $\beta_{i}, \beta_{c}$ and $\beta_{d}$ to be uncorrelated. Meanwhile, intuition suggests that $\phi_{i}^{*} \rightarrow \varphi_{i}^{*}$ as $\beta_{i} \rightarrow 1\left(\operatorname{Var}\left(n_{i}\right) \rightarrow 0\right)$. On the other hand, in practice, insureds are not allowed to select coverage levels larger than 1 . With the fact that $\beta_{i}$ will fluctuate around $81, \forall i=1,2, \ldots, n$ and some simulation results, in most cases, both $\phi_{i}^{*}$ and $\varphi_{i}^{*}$ will exceed 1 , thus driving most farmers choose to purchase a full coverage.

\section{A modified Miranda Decomposition}

DYNAMIC MODELS OF MIXED COVERAGE CROP YIELD INSURANCE

Going back, the Miranda Decomposition (1.1) is actually a static approach with predetermined $\mu_{i}$ and $\mu$ that constructs $y_{i}$ through the product of external random variables $y_{c}$ and $\epsilon_{i}$, therefore farmers' control towards their production activities are not reflected. In this case, we make some sight modifications on (1.1) to make it feasible of reflecting farmers' dynamic behaviors.

Based on (1.1), $y_{i}$ could be decomposed in a dynamic way, that is,

$$
\begin{aligned}
& y_{i t}=\mu_{i t}+k \beta_{i}\left(y_{c t}-\mu\right)+k \epsilon_{i t}, \\
& \text { where } E\left(y_{c t}\right)=\mu, \quad E\left(\epsilon_{i t}\right)=0, \quad k=\left\{\begin{array}{l}
\frac{\mu_{i t}}{M}, \quad \text { if } \mu_{i t}>0, \\
0, \quad \text { if } \mu_{i t}=0 .
\end{array}\right.
\end{aligned}
$$

The assumptions in (2.1) are analogous to those in (1.1) expect no longer assuming an timeinvariant $\mu_{i}$. In fact, $\mu_{i t}$ measures the willingness yield (or controllable yield) for farmer $i$ at time $t$, given all the information up to time $t$ (including premium and cost function, etc). 
Besides, $y_{c t}$ and $\epsilon_{i t}$ are still uncontrollable ${ }^{9}$ for farmer $i$ so that their expectations $\mu$ and 0 remain to be time-invariant. As a simple fact, the fluctuation of $y_{i t}-\mu_{i t}$ should be increasing with $\mu_{i t}$, therefore we further assume the distributions of $y_{c t}$ and $\epsilon_{i t}$ to be $k y_{c}^{M}$ and $k \epsilon^{M}$, where $y_{c}^{M}-\mu$ and $\epsilon^{M}$ refer to the standardized systemic and non-systemic fluctuations under input $M$. Moreover, $\beta_{i}$ is also time-invariant because we are not intend to model it due on the fact that it is not fully controllable for farmer $i$. With the help of (2.1), we could model the behavioral dynamics for an individual farmer under different insurance arrangements.

\section{Optimizing farmers' production dynamics under area-yield and individual-yield crop insurance}

Suppose $\mu_{i t}$ is the willingness yield for farmer $i$ at time $t$, the aggregated production cost for producing such amount is $H_{i}\left(W_{t}, \mu_{i t}\right)$ with $W_{t}$ denoting the external conditions at time $t$ systemically affecting all farmers' productions that will change the shape of $H$ as an adjuster. In nature, for any given $W_{t}, H$ should be a non-negative increasing function of $\mu_{i, t}$. For simplicity, we further assume $H_{i}\left(W_{t}, \mu_{i t}\right)$ to be strictly convex increasing ${ }^{10}$ with respect to $\mu_{i t}, \forall W_{t}$. So we have $\frac{\partial H_{i}\left(W_{t}, \mu_{i t}\right)}{\partial \mu_{i t}}>0$ and $\frac{\partial^{2} H_{i}\left(W_{t}, \mu_{i t}\right)}{\partial \mu_{i t}{ }^{2}}>0$. At this phase, we consider a fairly intuitive optimization criteria, which is, farmer $i$ decides his willingness yield $\mu_{i t}$ at time $t$ in a manner that maximizes his expected income-cost ratio $E\left[R_{i t}\left(W_{t}, \mu_{i t}\right)\right]=E\left[\frac{\operatorname{Income}\left(W_{t}, \mu_{i t}\right)}{\operatorname{Cost}\left(W_{t}, \mu_{i t}\right)}\right]$. Furthermore, under given ${ }^{11} W_{t}$ and fixed price per product $P$, if farmer $i$ purchases a full coverage level of area-yield crop insurance with indemnity $I_{i t}^{\text {area }}=n_{c t}=\left(x_{c t}-y_{c t}\right)_{+}$and premium $\pi_{i t}^{\text {area }}$, his optimal willingness yield is determined by

$$
\max _{\mu_{i t}} E\left[R_{i t}\left(\mu_{i t}\right)\right]=\max _{\mu_{i t}} E\left\{\frac{P\left[\mu_{i t}+k \beta_{i}\left(y_{c t}-\mu\right)+k \epsilon_{i t}+n_{c t}\right]}{H_{i}\left(\mu_{i t}\right)+\pi_{i t}^{\text {area }}}\right\}=\max _{\mu_{i t}}\left\{\frac{P\left[\mu_{i t}+E\left(n_{c t}\right)\right]}{H_{i}\left(\mu_{i t}\right)+\pi_{i t}^{\text {area }}}\right\} .
$$

Consequently ${ }^{12}$,

$$
\frac{\partial E\left[R_{i t}\left(\mu_{i t}\right)\right]}{\partial \mu_{i t}}=\frac{P\left\{H_{i}\left(\mu_{i t}\right)+\pi_{i t}^{\text {area }}-\left[\mu_{i t}+E\left(n_{c t}\right)\right] H_{i}^{\prime}\left(\mu_{i t}\right)\right\}}{\left[H_{i}\left(\mu_{i t}\right)+\pi_{i t}^{\text {area }}\right]^{2}},
$$

yields the first order condition to be

$$
H_{i}\left(\tilde{\mu}_{i t}\right)+\pi_{i t}^{a r e a}-\left[\tilde{\mu}_{i t}+E\left(n_{c t}\right)\right] H^{\prime}{ }_{i}\left(\tilde{\mu}_{i t}\right)=0
$$

So that

$$
\left.\frac{\left.\partial^{2} E\left[R_{i t}\right)\right]}{\partial \mu_{i t}{ }^{2}}\right|_{\mu_{i t}=\widetilde{\mu}_{i t}}=\frac{-\left[\widetilde{\mu}_{i t}+E\left(n_{c t}\right)\right]\left[H_{i}\left(\widetilde{\mu}_{i t}\right)+\pi_{i t}^{a r e a}\right]^{2} H^{\prime \prime} i\left(\widetilde{\mu}_{i t}\right)}{\left[H_{i}\left(\widetilde{\mu}_{i t}\right)+\pi_{i t}^{a r e a}\right]^{2}}<0,
$$

which infers an unique global maximum.

In order to extract mathematical conclusions, we assume $H_{i}\left(\mu_{i t}\right)$ to be an exponential function of $\mu_{i t}$, which is, $H_{i}\left(\mu_{i t}\right)=C_{i} e^{\gamma_{i} \mu_{i t}}$, with $C_{i}$ denoting the fixed cost for any willingness yield. Moreover, it is apparent that $\frac{H_{i}^{\prime}\left(\mu_{i t}\right)}{H_{i}\left(\mu_{i t}\right)}=\gamma_{i}$ which states that the growth rate of production cost with respect to willingness yield is time-invariant to a farmer while distinctive among farmers.

\footnotetext{
${ }^{9}$ Despite the fact that $y_{c}=\sum_{i=1}^{n} \omega_{i} y_{i}$, farmer $i$ makes nearly no influence on $y_{c}$, which is same for the cases if $y_{c}$ is replaced by weather index or other external random variables

10 strictly increasing marginal costs

11 in this case, $H_{i}\left(W_{i t}, \mu_{i t}\right)$ and $R_{i t}\left(W_{i t}, \mu_{i t}\right)$ are denoted as $H_{i}\left(\mu_{i t}\right)$ and $R_{i t}\left(\mu_{i t}\right)$

12 denote $H_{i}{ }^{\prime}(\cdot)$ and $H^{\prime \prime}{ }_{i}(\cdot)$ the first and second order differentials of $H_{i}(\cdot)$ with respect to $\mu_{i t}$
} 
Therefore, $\gamma_{i}$ could be regarded as a measure of production efficiency for farmer $i$. Together with $C_{i}$, we could compare the production costs within all farmers on a standardized basis. In this case, substituting $H_{i}\left(\mu_{i t}\right)=C_{i} e^{\gamma_{i} \mu_{i t}}$ into (2.3), the first order condition will be

$$
\begin{aligned}
& C_{i} e^{\gamma_{i} \widetilde{\mu}_{i t}}+\pi_{i t}^{a r e a}-\left[\tilde{\mu}_{i t}+E\left(n_{c t}\right)\right] C_{i} \gamma_{i} e^{\gamma_{i} \tilde{\mu}_{i t}}=0 \\
& \Leftrightarrow 1+\frac{\pi_{i t}^{a r e a}}{C_{i}}\left(e^{-\gamma_{i}}\right)^{\widetilde{\mu}_{i t}}=\left[\tilde{\mu}_{i t}+E\left(n_{c t}\right)\right] \gamma_{i} .
\end{aligned}
$$

Clearly, the left-side and right-side term of (2.4) could be regarded as an decreasing exponential function (given that $\gamma_{i}<1$ ) and an increasing linear function of $\tilde{\mu}_{i t}$ respectively, yields $\tilde{\mu}_{i t}$ to be positioned at the unique intersection point of these two functions. Together with the boundary condition $\mu_{i t} \geqslant 0$, we obtain

$$
\begin{gathered}
\max _{\mu_{i t}} E\left[R_{i t}\left(\mu_{i t}\right)\right]=E\left[R_{i t}\left(\mu_{i t}^{*}\right)\right]= \\
\left\{\begin{array}{l}
\left.\frac{P E\left(n_{c t}\right)}{C_{i}+\pi_{i t}^{\text {area }}}\right|_{\mu_{i t}^{*}=0}, \quad \text { if } 1+\frac{\pi_{i t}^{\text {area }}}{C_{i}} \leqslant E\left(n_{c t}\right) \gamma_{i}, \\
\left.\frac{P}{\gamma_{i} C_{i} e^{\gamma_{i} \tilde{\mu}_{i t}}}\right|_{\mu_{i t}^{*}=\widetilde{\mu}_{i t}>0,} \text { if } 1+\frac{\pi_{i t}^{\text {area }}}{C_{i}}>E\left(n_{c t}\right) \gamma_{i} .
\end{array}\right.
\end{gathered}
$$

Indeed, if farmer $i$ doesn't purchase any insurance, his optimal willingness yield $\mu_{i t}^{*}$ is simply determined by

$$
\max _{\mu_{i t}} E\left[R_{i t}\left(\mu_{i t}\right)\right]=\max _{\mu_{i t}} E\left\{\frac{P\left[\mu_{i t}+k \beta_{i}\left(y_{c t}-\mu\right)+k \epsilon_{i t}\right]}{H_{i}\left(\mu_{i t}\right)}\right\}=\max _{\mu_{i t}}\left\{\frac{P\left(\mu_{i t}\right)}{H_{i}\left(\mu_{i t}\right)}\right\} \Rightarrow \mu_{i t}^{*}=\frac{1}{\gamma_{i}}>0 .
$$

From (2.5), we know that if $1+\frac{\pi_{i t}^{\text {area }}}{c_{i}} \leqslant E\left(n_{c t}\right) \gamma_{i}$, the optimal willingness yield under area-yield insurance would be 0 , which is less than $\frac{1}{\gamma_{i}}$ for no insurance case. Though an individual farmer could not control the indemnity in area-yield insurance, but he could still reach the maximum expected income-cost ratio at willingness yield as 0 under certain circumstances. Therefore, we refer to this situation as "moral hazard" in area-yield insurance. On the other hand, if $1+$ $\frac{\pi_{i t}^{a r e a}}{c_{i}}>E\left(n_{c t}\right) \gamma_{i}$, farmer $i$ would purchase an area-yield insurance only when $\tilde{\mu}_{i t}$ extracted from (2.4) is less than $\frac{1}{\gamma_{i}}$. Because otherwise the maximum expected income-cost ratio will be less than that when he doesn't purchase insurance, since $E\left[R_{i t}\left(\mu_{i t}^{*}\right)\right]$ is decreasing with $\mu_{i t}^{*}$ in both cases. The case $\mu_{i t}^{*}=0$ may seem to be fairly abnormal in the first glance, but it makes sense if farmers are allowed to freely choose what they do to make income. Indeed, $E\left[R_{i t}\left(\mu_{i t}^{*}\right)\right]$ could be treated as the maximum rate of return in conducting agricultural production for farmer $i$ at time $t$. When $\mu_{i t}^{*}=0$, he could put the rest of his capital (and labor, time, etc) into relatively more profitable activities rather than agricultural production. Besides, the results we get are derived from relatively strong assumptions such as strictly convex increasing $\operatorname{cost}^{13}$ and ignorance of utility ${ }^{14}$, which may lack reliability in practices.

From the previous analysis, we obtain that farmer $i$ will purchase individual-yield insurance and reach a positive optimal willingness yield if and only if

\footnotetext{
13 in practice, cost functions are more common to be concave-convex function with potential inflection points ${ }^{14}$ we only consider " $E(R)$ " instead of " $E[U(R)]$ "
} 


$$
\begin{aligned}
& 1+\frac{\pi_{i t}^{\text {area }}}{C_{i}}>E\left(n_{c t}\right) \gamma_{i} \text { and } \tilde{\mu}_{i t}<\frac{1}{\gamma_{i}} \\
& \Leftrightarrow\left\{\begin{array}{l}
1+\frac{\pi_{i t}^{\text {area }}}{C_{i}}>E\left(n_{c t}\right) \gamma_{i} \\
H_{i}\left(\tilde{\mu}_{i t}\right)+\pi_{i t}^{\text {area }}-\left.\left[\tilde{\mu}_{i t}+E\left(n_{c t}\right)\right] H^{\prime}{ }_{i}\left(\tilde{\mu}_{i t}\right)\right|_{\tilde{\mu}_{i t}=\frac{1}{\gamma_{i}}}<0
\end{array}\right. \\
& \Leftrightarrow \quad \frac{\pi_{i t}^{\text {area }}}{C_{i} e}<E\left(n_{c t}\right) \gamma_{i}<1+\frac{\pi_{i t}^{\text {area }}}{C_{i}}
\end{aligned}
$$

which yields the following proposition.

Proposition 2.1 Under area-yield coverage arrangement, farmer $i$ will purchase insurance and reach a positive optimal willingness yield only when $\frac{\pi_{i t}^{\text {area }}}{C_{i} e}<E\left(n_{c t}\right) \gamma_{i}<1+\frac{\pi_{i t}^{\text {area }}}{C_{i}}$, otherwise he would either quit insurance $\left(\mu_{i t}^{*}>\frac{1}{\gamma_{i}}\right)$ or act in a moral hazard manner $\left(\mu_{i t}^{*}=0\right)$.

Moreover, if farmer $i$ purchases a full coverage of individual-yield crop insurance with indemnity $I_{i t}^{\text {ind }}$ and premium $\pi_{i t}^{i n d}$, we still intend to use the same method as in above to find out his optimal willingness yield. Practically, the indemnity for individual-yield crop insurance is usually of the form $I_{i t}^{\text {ind }}=\left(a_{i t}-y_{i t}\right)_{+}$with $a_{i t}$ as its threshold. Yet mathematically, $E\left(I_{i t}^{\text {ind }}\right)=$ $E\left[a_{i t}-\mu_{i t}-k \beta_{i}\left(y_{c t}-\mu\right)-k \epsilon_{i t}\right]_{+}$composed of a non-random component $\mu_{i t}$ and a random component $k \beta_{i}\left(y_{c t}-\mu\right)+k \epsilon_{i t}$ could not be further decomposed ${ }^{15}$, which makes the optimization of $E\left[R_{i t}\left(\mu_{i t}\right)\right]$ infeasible. In this case, we implement a special form that $I_{i t}^{\text {ind }}=$ $\left(a_{i t}-\mu_{i t}\right)_{+}$with the rest random terms of $y_{i t}$ dropped in order to emphasize the greater control of farmer $i$ towards his indemnity payment under individual-yield crop insurance arrangement. Hence, the optimal willingness yield $\mu_{i t}^{*}$ for farmer $i$ at time $t$ under a full coverage level ${ }^{16}$ of individual-yield crop insurance is determined by

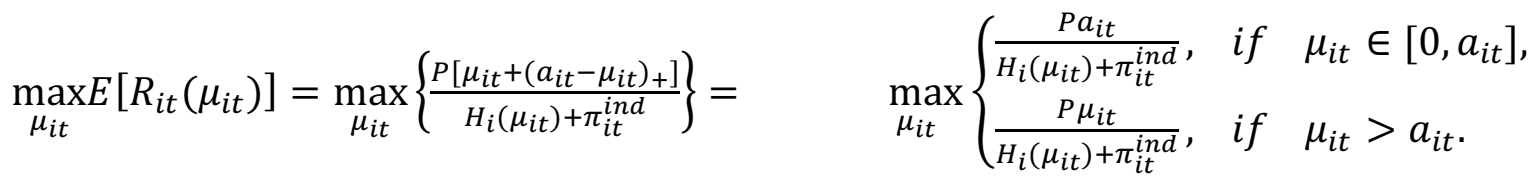

From (2.7), we know that

$$
\left.\frac{\partial E\left[R_{i t}\left(\mu_{i t}\right)\right]}{\partial \mu_{i t}}\right|_{\mu_{i t} \in\left[0, a_{i t}\right]}=\frac{-P a_{i t} H_{i}^{\prime}\left(\mu_{i t}\right)}{\left[H_{i}\left(\mu_{i t}\right)+\pi_{i t}^{i n d}\right]^{2}}<0 .
$$

Moreover,

$$
\begin{gathered}
\left.\frac{\partial E\left[R_{i t}\left(\mu_{i t}\right)\right]}{\partial \mu_{i t}}\right|_{\mu_{i t}>a_{i t}}=\frac{P\left[H_{i}\left(\mu_{i t}\right)+\pi_{i t}^{i n d}-\mu_{i t} H_{i}\left(\mu_{i t}\right)\right]}{\left[H_{i}\left(\mu_{i t}\right)+\pi_{i t}^{i n d}\right]^{2}} \\
\Rightarrow H_{i}\left(\tilde{\mu}_{i t}\right)+\pi_{i t}^{i n d}=\tilde{\mu}_{i t} H^{\prime}{ }_{i}\left(\tilde{\mu}_{i t}\right) \\
\left.\Rightarrow \frac{\partial^{2} E[R i t}{\partial \mu_{i t}{ }^{2}}\right|_{\mu_{i t}=\widetilde{\mu}_{i t}}=\frac{-P \widetilde{\mu}_{i t} H^{\prime \prime}\left({ }^{\prime}\left(\widetilde{\mu}_{i t}\right)\left[H_{i}\left(\widetilde{\mu}_{i t}\right)+\pi_{i t}^{i n d}\right]^{2}\right.}{\left[H_{i}\left(\widetilde{\mu}_{i t}+\pi_{i t}^{i n d}\right)\right]^{4}}<0
\end{gathered}
$$

infers an unique local maximum. Therefore, by (2.8), (2.9) and (2.10), it is obtained that

\footnotetext{
15 because $(a+b)_{+} \neq a_{+}+b_{+}$

16 note that the variance reduction of individual-yield crop insurance is increasing with coverage level, thus leading full coverage to be optimal
} 


$$
\begin{gathered}
\max _{\mu_{i t}} E\left[R_{i t}\left(\mu_{i t}\right)\right]=E\left[R_{i t}\left(\mu_{i t}^{*}\right)\right]= \\
\left\{\begin{array}{l}
\left.\frac{P a_{i t}}{C_{i}+\pi_{i t}^{i n d}}\right|_{\mu_{i t}^{*}=0} ^{*}, \quad \text { if } \quad \frac{P}{n_{i} C_{i} e^{\gamma_{i} \tilde{\mu}_{i t}}} \leqslant \frac{P a_{i t}}{\pi_{i t}^{i n d}+C_{i}} \quad \text { or } \quad \tilde{\mu}_{i t} \leqslant a_{i t}, \\
\left.\frac{P}{\gamma_{i} C_{i} e^{\gamma_{i} \tilde{\mu}_{i t}}}\right|_{\mu_{i t}^{*}=\widetilde{\mu}_{i t}}, \quad \text { if } \quad \frac{P}{\gamma_{i} C_{i} e^{\gamma_{i} \tilde{\mu}_{i t}}}>\frac{P a_{i t}}{\pi_{i t}^{i n d}+C_{i}}, \quad \tilde{\mu}_{i t}>a_{i t} .
\end{array}\right.
\end{gathered}
$$

From (2.11), we know that under individual-yield insurance arrangement, farmer $i$ could reach an maximum expected income-cost ratio at a non-negative willingness yield only if $\mu_{i t}^{*}$ equals to $\tilde{\mu}_{i t}$ derived from (2.10). Hereby, substituting $\tilde{\mu}_{i t}=\frac{1}{\gamma_{i}}$ into (2.10), we have

$$
H_{i}\left(\tilde{\mu}_{i t}\right)+\pi_{i t}^{i n d}-\left.\tilde{\mu}_{i t} H^{\prime}{ }_{i}\left(\tilde{\mu}_{i t}\right)\right|_{\widetilde{\mu}_{i t}=\frac{1}{\gamma_{i}}}=\frac{\pi_{i t}^{i n d}}{C_{i} e}>0 .
$$

Consequently, (2.12) indicates that farmer $i$ will not choose a positive $\mu_{i t}^{*}$ under individualyield coverage, because he needs to acquire larger optimal willingness yield while obtaining less expected income-cost ratio compared with the no insurance case. Therefore, the following proposition holds.

Theorem 2.2 Under individual-yield coverage arrangement, moral hazard will bound to occur $\left(\mu_{i t}^{*}=0\right)$ if farmer $i$ choose to purchase an insurance. Therefore, moral hazard in individual-yield insurance is far more severe than that in area-yield insurance.

\section{References}

Chambers, Robert G. "Insurability and Moral Hazard in Agricultural Insurance Markets." Amer. Agr. Econ. 71(1989):604-16.

Barnett, B. J., J. R. Black, Y. Hu, and J. R. Skees. Is Area Yield Insurance Competitive with Farm Yield Insurance? Journal of Agricultural and Resource Economics. 30(2005):285-301.

Bulut, H., K. J. Collins, and T. P. Zacharias. Optimal Coverage Level Choice with Individual and Area Insurance Plans. American Journal of Agricultural Economics. 94(2012):1013-1023

Miranda, M. J. Area-Yield Crop Insurance Reconsidered. American Journal of Agricultural Economics 73(1991):233242 